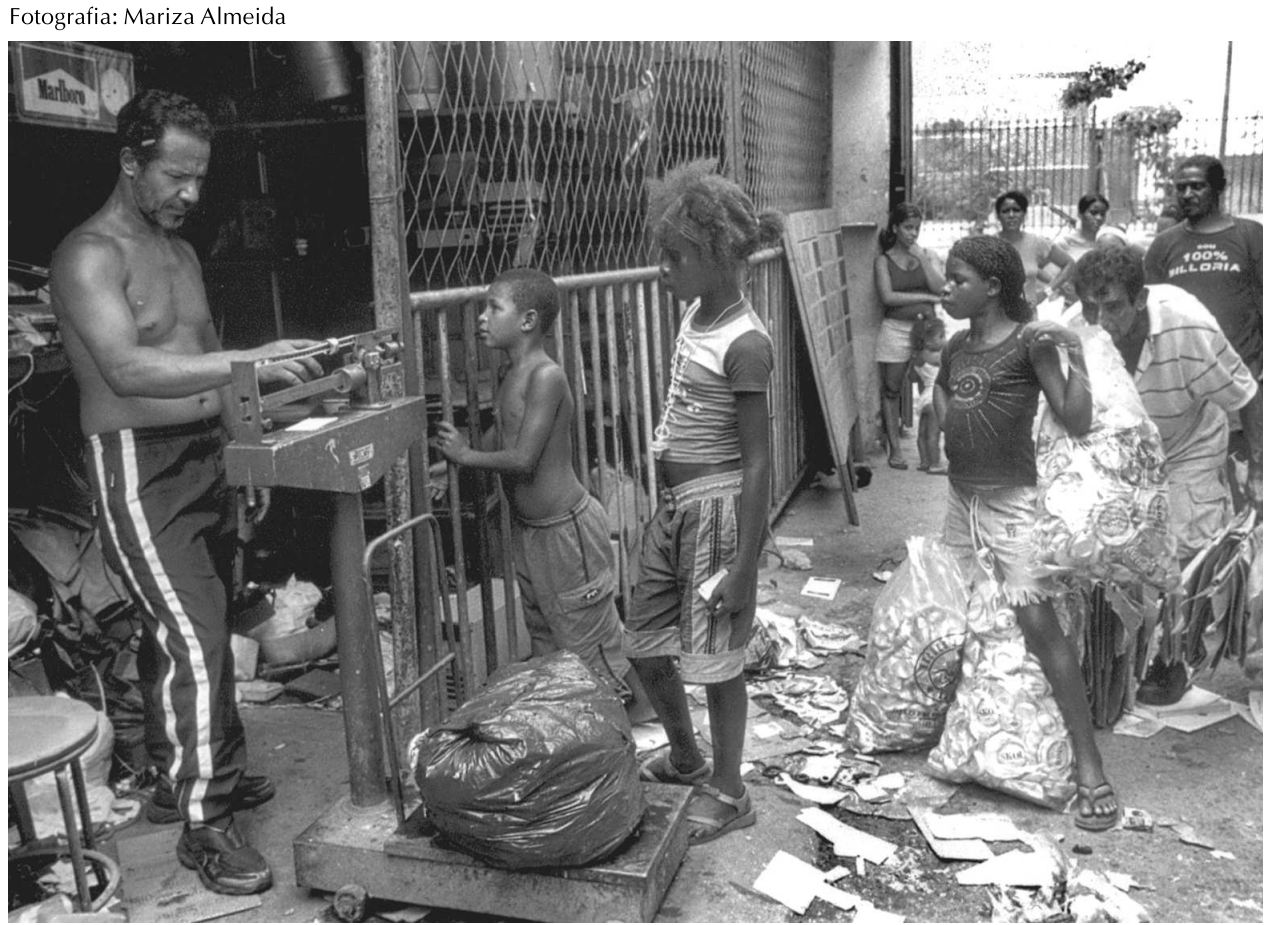




\section{Marina Capello* Sergio Gianna** \\ Emancipación política y emancipación humana: un debate necesario sobre ciudadanía a partir de Marx}

Emancipação política e emancipação humana: um debate necessário sobre cidadania a partir de Marx

Political emancipation and human emancipation: a necessary debate on citizenship based on Marx

Resumen: Este artículo tiene por finalidad el análisis de la dialéctica entre mancipación política y la emancipación humana, centralmente a partir de la obra "Acerca de la Cuestión Judía" de Marx, texto relegado dentro del debate marxista en Argentina. Desde la comprensión de los límites de la ciudadanía y la emancipación política, es posible polemizar con las obras de Marshall y Sousa Santos, teóricos que en diversos momentos históricos estudian la temática de la ciudadanía y que en la actualidad, son las dos fuentes principales en los autores argentinos que piensan la relación entre el Trabajo Social y la ciudadanía.

Palabras claves: emancipación política, emancipación humana, ciudadanía, trabajo social.

Resumo: O presente artigo tem como finalidade analisar a dialética entre emancipação política e emancipação humana, centralmente a partir da obra "Sobre a questão judaica" de Marx, texto relegado no debate marxista na Argentina. A partir da compreensão dos limites da cidadania e da emancipação política, é possível polemizar com as obras de Marshall e Sousa Santos, teóricos que em diversos momentos históricos estudam a temática da cidadania e que, na atualidade, são as duas fontes principais dos autores argentinos que pensam a relação entre Serviço Social e cidadania.

Palavras-chave: emancipação política, emancipação humana, cidadania, serviço social.

Abstract: This article aims to analyze the dialectic between political emancipation and human emancipation, centrally from the book "On the Jewish Question" neglected text within the Marxist debate in Argentina. From understanding the limits of citizenship and political emancipation, it is possible to argue with the works of Marshall and Sousa Santos, theorists that in various historical moments have studied the issue of citizenship and currently are the two main sources of Argentine authors who think the relationship between social work and citizenship.

Keyword: political emancipation, human emancipation, citizenship, social work.

\footnotetext{
* Licenciada en Trabajo Social. Profesora de la Cátedra Trabajo Social V, Investigadora y Extensionista (FTS UNLP). Miembro del Instituto de Capacitaciones de Estudios Profesionales (ICEP) del CPTS de Pcia. de Bs. As. Email: marinacapello@yahoo.com.ar.

** Licenciado en Trabajo Social (ETS-UNC). Magister en Trabajo Social (FTS-UNLP) y Doctorando en Ciencias Sociales (FCS-UBA). Docente adscripto del a materia "Epistemología de las Ciencias Sociales" (FTS-UNLP) y Becario de CONICET. E-mail: sdgianna@gmail.com.
} 


\section{nevista Q all pallat}

\} EMANCIPACIÓN POLÍTICA Y EMANCIPACIÓN HUMANA - CAPELO, M.; GIANNA, S. \}

\section{Introducción}

El presente artículo, tiene por objetivo analizar a partir de la obra de Marx, Acerca de la Cuestión Judía - publicado en los Anales Franco-Alemanes en Paris, en 1844 - las categorías emancipación política y emancipación humana en dialogo o contrapunto con las categorías de ciudadanía y democracia que imperan con sostenida hegemonía en la teoría social y cuyo impacto ha crecido en los últimos años en el Trabajo Social argentino.

En un primer momento, se analizará en profundidad las categorías de emancipación política y emancipación humana que Marx presenta a la luz del debate filosófico-político que sostiene con Bruno Bauer, representante del idealismo hegeliano de su época. Se expondrá cómo este texto contiene ya las premisas para analizar la realidad desde el materialismo histórico, dando cuenta, en un primer esbozo, de la escisión que presenta la sociedad burguesa, cuando se configura la unidad contradictoria entre Sociedad Civil y Estado, a partir del específico y particular modo de producción capitalista. En este apartado, también se recuperará las posiciones teóricas de Marshall y Sousa Santos, quienes en los últimos años han venido discutiendo en torno a las categorías de ciudadanía y emancipación y hoy, pueden ser considerados dos de los referentes centrales en torno a esta temática, tanto para las ciencias sociales como el Trabajo Social.

En un segundo momento, el trabajo abordará la influencia de la categoría ciudadanía en el debate contemporáneo del Trabajo Social argentino. En este apartado, se buscará analizar la aparición de la categoría ciudadanía y emancipación política en diversas instancias de la profesión, tanto en lo normativo, en los procesos cotidianos de intervención profesional y en el debate teórico. La recuperación en el primer momento de las obras de Marshall y Sousa Santos, remite a que estos autores han incidido en las discusiones profesionales en torno a la ciudadanía, al mismo tiempo que es Ilamativa la escasa presencia de producciones provenientes de la tradición marxiana y marxista que aborden específicamente dicha temática.

\section{Dialéctica de la emancipación política y emancipación humana 1.1 La emancipación humana en Marx}

Hoy sabemos ya que ese reino de la razón no era más que el reino idealizado de la burguesía; que la justicia eterna vino a tomar cuerpo en la justicia burguesa; que la igualdad se redujo a la igualdad burguesa ante la ley; que como uno de los derechos más esenciales del hombre se proclamó la propiedad burguesa. (F. ENGELS).

La obra Acerca de la Cuestión Judía fue publicada en febrero de 1844 en los Anales Franco-Alemanes. La misma, es parte de una polémica más amplia sostenida por Marx y Engels con los hegelianos de izquierda, en particular, con su representante más destacado, Bruno Bauer. La crítica que realiza Marx y Engels a los hegelianos de izquierda es que estos toman con superficialidad las ideas comunistas, utilizándolas como mera fraseología, al mismo tiempo que ponen en evidencia la inmadurez 


\section{heVibtg am pant?}

\} EMANCIPACIÓN POLÍTICA Y EMANCIPACIÓN HUMANA - CAPELO, M.; GIANNA, S. \}

política de dicho grupo, al limitar sus críticas a la religión y a la difusión del ateísmo, desatendiendo las tareas centrales de la lucha contra el absolutismo feudal y los temas acuciantes provenientes de los problemas sociales. Al respecto de la ruptura de Marx con los hegelianos de izquierda, este señala en una de sus cartas que le dirigió a Ruge:

[...] pues deseaba más criticar la religión en la crítica de las circunstancias políticas que las circunstancias políticas en la religión, pues la religión que en si carece de contenido, no viene del cielo, sino de la tierra, y se derrumba por si sola, en cuanto se disuelve la realidad invertida, cuya teoría es la religión. (LUKÁCS, 2005, p. 130).

El texto Acerca de la Cuestión Judía es la crítica que Marx realiza a los textos de Bauer sobre la situación de la emancipación política de los judíos en Alemania, quienes en ese momento se encontraban aislados al igual que en el período del Antiguo Régimen y debiéndose refugiar en actividades comerciales y usureras, pese a que la Constitución Francesa de 1789 les abrió la posibilidad de su pertenencia como ciudadanos. Es así como los judíos alemanes no poseían los mismos derechos que los demás ciudadanos, por no profesar la religión del Estado alemán'.

En este contexto histórico, Bauer explicó que en Alemania nadie se encontraba emancipado políticamente en lo que atañe al carácter religioso del Estado, que solo reconocía privilegios. Por lo tanto, la lucha específica por la emancipación del judío debería expresarse como lucha general de la emancipación política de los alemanes. En palabras de Bauer "[...] El judío no debe ser emancipado por ser judío, en virtud de tener excelente principio humano y universal moralidad. El judío debe antes retirarse para atrás del ciudadano y ser un ciudadano aunque sea o desee ser un judío." (MARX, 2008, p. 173).

Por ello, Bauer sostuvo la necesidad de transformar el Estado Cristiano en un Estado Laico (democrático-burgués), identificando la tensión proveniente del condicionamiento motivado tanto por la esencia que porta el judío como por la esencia que porta el Estado Cristiano, en donde ambos son incapaces de conceder y propiciar la emancipación política. Llegando así a la reflexión de que sólo la emancipación del Estado respecto de la religión garantizaría que los individuos, sin obligación de una religión oficial, asumirían en igualdad las condiciones de ciudadano, escindiendo así, la esfera pública de la privada. Concibiendo el ámbito de lo privado como el espacio por excelencia donde los individuos puedan o se sientan obligados a cumplir sus deberes religiosos. Subyace ya en este análisis lo público condensado en lo estatal y lo privado condensado en la sociedad civil.

\footnotetext{
1 Se recuerda que Alemania se caracterizaba por ser el país más atrasado en sus condiciones económicas políticas, donde el poder político aun lo tenía la Monarquía, bajo el trono de Federico Guillermo IV de Prusia, quien al recrudecer en su política interior las medidas restauradoras, agudizó un clima de conflicto social, abriéndose así un periodo de luchas, de las cuales participó una nueva pero pujante clase obrera, que culminó con la revolución democrática-burguesa de 1848. 


\section{heVibtg am pant?}

\} EMANCIPACIÓN POLÍTICA Y EMANCIPACIÓN HUMANA - CAPELO, M.; GIANNA, S. \}

Para Marx, los límites de la emancipación política no se reducen a la emancipación de la religión, ya que en sí misma no resuelve las contradicciones de la emancipación humana, contradicciones que atraviesan al hombre egoísta, al hombre como ser social pero no al ciudadano. Que el Estado pueda liberarse de la religión, no da cuenta de que el hombre real se libere de ella. Siguiendo este pensamiento, Marx concluye que la religión posicionada dentro de la sociedad burguesa es el ejemplo fantasioso de las relaciones sociales, una forma particular que impide que los seres humanos se encuentren a sí mismos como sujetos de la historia humana, en donde la conciencia de Dios es la conciencia que el ser humano tiene de sí mismo, proyectada para y hacia afuera.

La emancipación política (que contiene a la emancipación religiosa) expresa la relación entre sociedad burguesa y Estado, en la cual el Estado se constituye como una universalidad por encima de los elementos particulares. Marx critica que Bauer plantea la cuestión en términos idealistas, religiosos y teológicos ya que reproduce abstractamente y con menos riqueza el concepto filosófico de esencia reducido solo a la religión, ecualizando así emancipación política con emancipación religiosa, considerando que solo renunciando a ser judío se lograría la emancipación política. Por lo tanto, la emancipación del judío sólo se realiza si se niega a sí mismo.

En la crítica a Bauer subyace la crítica materialista a la dialéctica hegeliana. Marx invierte en forma materialista la relación entre sociedad burguesa y Estado. Retoma el concepto de Hegel de sociedad civil pero como el punto de partida, como la verdadera trama en donde se originan y producen las distintas formas de Estado y sus relaciones jurídicas. Su crítica cuestiona la autonomización del concepto y la exageración idealista de las abstracciones, que las transforma en esencias autónomas, poniendo sobre los pies la filosofía hegeliana. Ya no es la idea del Estado como razón universal la que produce a la sociedad burguesa, sino que la sociedad burguesa es la condición para la existencia del Estado político, el propio Estado es producto y contiene las contradicciones de la propia sociedad civil. En este sentido se debe considerar a la sociedad civil como el genuino escenario donde se desarrolla la lucha de clases.

En su crítica a Bruno Bauer, Marx desenmascara la contradicción interna de la sociedad burguesa, introduciendo de otro modo el interrogante sobre la emancipación ${ }^{2}$. Su pregunta no es sobre quién será emancipado, sino qué clase de emancipación está en juego (MARX, 2008). Mediante esta pregunta, Marx revela la diferencia fundamental entre la emancipación política y la emancipación humana: la política es un avance, un progreso dentro del orden social vigente, en cambio la emancipación humana presupone la superación de la "autoalienación humana", un orden social donde las contradicciones de clase se resuelvan y superen.

Para Marx, la emancipación política crea una democracia formal que promulga derechos y libertades que no pueden existir concretamente en la sociedad

\footnotetext{
${ }^{2}$ En este planteo se encuentra el germen de su concepción dialéctico-materialista de la historia, aunque todavía no haya desarrollado las categorías de la economía política que dan cuenta del modo de producción capitalista y el rol histórico del proletariado, como sujeto de la emancipación humana. En Sobre la Cuestión Judía, aun con un lastre feuerbachiano, pone en cuestión los elementos esenciales del liberalismo, llegando a un nudo importante a resolver, la contradicción entre el derecho formal y su negación real, la crítica del dinero y los fundamentos de la sociedad civil. 


\section{nevistg am pguth}

\} EMANCIPACIÓN POLITICA Y EMANCIPACIÓN HUMANA - CAPELO, M.; GIANNA, S. \}

burguesa. La igualdad ante la ley anula políticamente las relaciones de propiedad de la sociedad burguesa, Marx reflexiona con aguda profundidad sobre este punto complejo y contradictorio, el avance de la ampliación de ciudadanía con el sufragio universal anulando el voto censitario, contiene como negación, la naturalización de la propiedad privada, ya que no soóo no la supera sino que también la da por supuesta.

La contraposición entre Estado Político y sociedad civil hace que el individuo además de ser reconocido en el Estado como ser genérico, caiga en la ilusión de que se torna genérico gracias al Estado, despojándolo del carácter colectivo de su vida material en nombre de una universalidad irreal; burgués (bourgeois) como miembro de la sociedad civil y ciudadano (citoyen) como individuo con derechos políticos en relación con el Estado. De aquí se deduce que la relación entre el Estado Político y la sociedad burguesa es espiritualista, escindiendo a cada hombre individual: citoyen - dimensión espiritualista y bourgeois - dimensión material.

A partir de esta escisión del individuo como citoyen y bourgeois, Marx coloca en su justo lugar el problema de la religión en la cuestión judía

La diferencia entre el hombre religioso y el ciudadano es la diferencia existente entre el comerciante y el ciudadano, entre el jornalero y el ciudadano, entre el individuo vivo y el ciudadano. La contradicción en que el hombre religioso se encuentra con el hombre político, es la misma en que se encuentra el bourgeois con el citoyen, con el que se encuentra el miembro de la sociedad burguesa con su piel de león política (MARX, 2008, p. 198).

Recuperando las constituciones de las diversas revoluciones burguesas, Marx demuestra que en las mismas el "homme" se diferencia y es subordinado al "citoyen". Desde esta línea de análisis, la emancipación política no resuelve dicho desgarramiento y escisión, peor aún, lo lleva a su extremo. Dirá Marx:

El hombre no fue liberado, pues, de la religión, sino que adquiere la libertad de religión. No fue liberado de la propiedad. Adquiere la libertad de propiedad. No fue liberado del egoísmo del oficio, sino que adquiere la libertad de oficio. Por lo tanto la sociedad burguesa se configura como el mundo de la propiedad privada en la que todo se convierte en mercancía [...]. (2008, p. 134).

La revolución política burguesa, en su propia constitución material no puede dar cuenta de la emancipación humana, por sus propias contradicciones entre los intereses de clase, que al no abolir la propiedad privada, persiste como sistema capitalista. Para lasi:

[...] el carácter genérico del ser humano en la igualdad ante la ley, mediada por el Estado, es la expresión de la universalidad del capital. Es el mejor de los artilugios para convalidar que no hay contradicción en los términos que expresan 


\section{heVibtg am pant?}

\} EMANCIPACIÓN POLÍTICA Y EMANCIPACIÓN HUMANA - CAPELO, M.; GIANNA, S. \}

esa igualdad: somos todos ciudadanos, miembros de la sociedad burguesa - o civil - somos todos, por tanto capital. Esa universalidad esconde el hecho de que la igualdad exige que algunos asuman el papel de acumuladores de plusvalía, en cuanto otros se transforman en mercancía, mientras que una vez consumida se transforman en capital. (2007, p.56).

Es decir, entre la ciudadanía y el modo en que se organiza el proceso de metabolismo social existen un conjunto de mediaciones que deben ser dilucidadas y para poder captar cuál es el lugar de la primera dentro del modo de producción capitalista, se vuelve necesario comprenderla como un momento de esa totalidad, buscando en ella su génesis y no, como en general la teoría política hace, buscar en una dimensión jurídico-política las bases de su legitimidad. Los fundamentos de la ciudadanía se encuentran en el modo histórico en el que el hombre organiza el proceso de producción y reproducción de la vida humana.

Por lo tanto, los derechos que Marx analizó en Acerca de la Cuestión Judía son los que hoy se conocen como los derechos civiles, siendo derechos de los individuosciudadanos que pueden ejercer contra las arbitrariedades del Estado (COUTINHO, 2000). ¿Cuáles son estos derechos? La libertad, la igualdad, la seguridad y la propiedad. Como recalca Marx (2008), el primero de estos derechos reconoce el poder del individuo de hacer todo lo que no perjudique a otros, la igualdad declara a todo individuo como libre, la seguridad busca preservar al individuo, sus derechos y propiedades y la propiedad asegurar y preservar el disfrute de los propios bienes, ingresos y frutos del trabajo.

Entre la democracia formal, conformada por los derechos de ciudadanía y el modo de producción se produce la mediación entre ciudadanos libres e iguales que intercambian sus mercancías a partir de relaciones jurídicas, esto es, intercambios concientes y voluntarios que no se basan en la violencia (MARX, 1971). Pero en el contenido de dicha relación se esconde la existencia de las clases sociales, ya que ciertos ciudadanos personifican en la "esfera privada" o Sociedad Civil al capital, bus-cando el interés egoísta y privado de la acumulación de capital y otros ciudadanos, personifican en la Sociedad Civil a la clase trabajadora, que deben vender su fuerza de trabajo para reproducir su vida.

Tanto la ciudadanía como la democracia formal desarrollada en el capitalismo, generan la apariencia de que han sido superadas las relaciones de explotación entre los hombres, mediante la abolición de la esclavitud y de los vínculos de dependencia personal y al mismo tiempo, porque el proceso de metabolismo social es organizado a partir de la cooperación entre individuos que "libremente" venden su fuerza de trabajo y otros individuos "libremente" la compran y consumen. Saliendo de la "forma celestial" de la ciudadanía y adentrándose en la "forma terrenal y profana" de la sociedad civil, la explotación del hombre sobre el hombre, la explotación de la fuerza de trabajo aún perdura, siendo el trabajo alienado el contenido del trabajo libre. Esta dualidad, es expresada por Marx con claridad al señalar que

El Estado suprime a su modo las diferencias de nacimiento, estamento, cultura, ocupación, declarándolas apolíticas, procla- 


\section{ApVista all piuth}

\} EMANCIPACIÓN POLIITICA Y EMANCIPACIÓN HUMANA - CAPELO, M.; GIANNA, S. \}

mando por igual a cada miembro del pueblo partícipe de la soberanía popular sin atender a esas diferencias, tratando a todos los elementos de la vida real del pueblo desde el punto de vista del Estado. No obstante, el Estado deja que la propiedad privada, la cultura, las ocupaciones actúen a su modo y hagan valer su ser específico. Muy lejos de suprimir estas diferencias de hecho, la existencia del Estado las presupone, necesita oponerse a estos elementos suyos para sentirse como Estado político e imponer su generalidad. (2006, p. 34).

Esta forma histórica que adquiere el modo de organización del proceso de metabolismo social lleva de modo inherente en sí mismo una creciente alienación del trabajo y de cosificación de las relaciones sociales. Esto, es justamente lo que permite ocultar la democracia formal: las relaciones de explotación y de desigualdad real, la comprensión del capitalismo como una sociedad de clases con intereses antagónicos entre la clase trabajadora y la burguesía.

De este modo, la emancipación política puede ser entendida como una democracia formal compatible con el capitalismo, que plantea una división entre una esfera pública secundada por el Estado y una esfera privada regida por la propiedad capitalista, donde la esfera política se encuentra separada de la económica, disociando el accionar del individuo como ciudadano y como propietario privado. Esto genera, que el interés general no sea más que la suma de los intereses particulares y que el Estado apenas sea el garante de los intereses de los productores que personifican al capital.

A diferencia de la emancipación política, la emancipación humana establece un modo de organizar el proceso de metabolismo social que acaba con las clases sociales y las relaciones entre personificaciones, produciendo una unidad inmediata y directa en el trabajo social, donde no se genera una producción por encima del tamaño de la necesidad social ni existan restricciones al acceso a los valores de uso productos del trabajo humano. Es decir, la emancipación humana genera un nuevo tipo de sociabilidad humana a partir de la transformación del proceso de trabajo, que deja de ser un trabajo privado que debe ser vendido en el mercado, para ser un trabajo de carácter asociado, directamente social, tanto en la producción, la circulación y el consumo.

Es por ello que para Marx aún se encuentra pendiente la emancipación humana, que no es más que una revolución social radical, no política. En sus propias palabras:

Toda emancipación es la recuperación del mundo humano, de sus relaciones, al hombre mismo. La emancipación política es la reducción del hombre de una parte a miembro de la sociedad Burguesa, al individuo egoísta independiente y de otra, al ciudadano del Estado, a la persona moral. Solo cuando el real hombre individual recupera en si al ciudadano abstracto, y como hombre individual se convierte en ser social en su vida empírica, en su 


\section{novigta am paltg}

\} EMANCIPACIÓN POLÍTICA Y EMANCIPACIÓN HUMANA - CAPELO, M.; GIANNA, S. \}

trabajo individual y en sus relaciones individuales, sólo cuando el hombre reconoce y organiza como sus forces propres como fuerzas sociales y cuando, por tanto, no se separa ya de sí la fuerza social en la forma de fuerza política, solo entonces se lleva a cabo la emancipación humana (2008, p. 197).

\subsection{Dialogando con Marx: ciudadanía y emancipación social - crítica de posiciones teóricas ancladas en la emancipación política \\ 1.2.1 La ciudadanía en Marshall}

La obra de Marx logra develar la dialéctica de la emancipación política y la emancipación humana, captando sus mediaciones y reconociendo que el ser genérico del hombre solo podrá realizarse con la superación de la emancipación política. En contraparte, otros autores provenientes de las ciencias sociales específicas, como Marshall y Sousa Santos, toman como objeto de análisis la cuestión de la ciudadanía y la emancipación como una de las posibles respuestas a las crisis cíclicas y estructurales que el capitalismo tuvo históricamente y actualmente porta. Se retoma estos dos autores, porque proponen una alternativa teórica y antagónica a la obra de Marx y por su importante influencia en el Trabajo Social argentino.

Thomas Marshall es uno de los mayores exponentes teóricos dentro del pensamiento de la sociología clásica, su preocupación como cientista social remite a la problemática social que acontece en la sociedad moderna, en el capitalismo contemporáneo y sus repetitivas crisis (específicamente de la post-Segunda Guerra Mundial, 1950), principalmente, ante la situación de la clase trabajadora, que nuevamente se organiza e interpela al orden vigente, denunciando en diversos conflictos la desigualdad social. En su libro, titulado Ciudadanía y Clase Social plantea que la ciudadanía se constituye en un sistema contrapuesto a las clases sociales. El autor, define a la ciudadanía como

[...] una condición otorgada a aquellos que son miembros plenos de una comunidad. Todos los que poseen la condición son iguales con respecto a los derechos y deberes con que está dotada esa condición... La clase social, por otra parte, es un sistema de desigualdad. Y también como la ciudadanía puede basarse en un conjunto de ideales, creencias y valores (MARSHALL, 2005, p. 37).

En este sentido, la categoría de ciudadanía impacta sobre las clases sociales, entendiendo que la "desigualdad social tiene un fin" (MARSHALL, 2005, p. 39), planteando así que

Los derechos de que estaba dotada la condición general de ciudadanía fueron extraídos del sistema de condición jerárquico de la clase social, despojándola de su sustancia esencial. La igualdad implícita en el concepto de ciudadanía, aunque limitada en contenido, socavaba la desigualdad del sistema de clases, que era 


\section{ApVista all piuth}

\} EMANCIPACIÓN POLÍTICA Y EMANCIPACIÓN HUMANA - CAPELO, M.; GIANNA, S. \}

en principio una desigualdad total. La justicia nacional y una ley común a todos inevitablemente debían debilitar y destruir la justicia de clase (MARSHALL, 2005, p. 38).

Desde esta perspectiva se aborda la desigualdad social pero desde la necesidad de convalidar y garantizar el funcionamiento y la estructura de la sociedad mediante una disminución de esta desigualdad; expresada en una preocupación por el futuro de la clase trabajadora, buscando preservarla para garantizar su reproducción como fuerza de trabajo, disciplinándola desde los propios deberes ciudadanos, "[...] proponiendo así reducir el volumen del trabajo para aquellos obreros que hacen trabajos pesados y excesivos, como así valorar la educación y el ocio" (MARSHALL, 2005, p.16).

Así se va constituyendo la ciudadanía moderna, que universaliza los derechos, que se expresan en su carácter formal y normativo aunque se revelen en sus múltiples aspectos, en sus elementos civiles, políticos y sociales ${ }^{3}$.

Lo que subyace en el pensamiento de Marshall resguarda una matriz liberal, desde una concepción ideal-liberal de la sociedad política, concediendo prioridad al concepto de individuo, reconociendo su pertenencia a una determinada comunidad o sociedad; marcando la importancia de su participación, que dé cuenta de su conciencia cívica; su necesario disciplinamiento, entendiendo que debe responder - como sujeto político o como sujeto de derecho - a deberes y obligaciones propios de la sociedad moderna.

Dicha concepción aborda los efectos de la distribución de la riqueza dentro de los parámetros del propio sistema, su estructura formal no toca la producción económica, la ciudadanía tiene una función integradora, requiere garantizar un vínculo armonioso entre las diferentes clases sociales. Son así necesarios para el mantenimiento de esta forma particular de desigualdad, por ejemplo los derechos civiles son indispensables en una competitiva economía de mercado, le dan a cada hombre condición individual para poder participar como unidad independiente en la lucha económica.

Al decir de Marshall, el contrato moderno es un acuerdo entre los hombres que son libres e iguales en su condición (no necesariamente en su poder), "[...] la desigualdad del sistema de clases sociales puede ser aceptable siempre que se reconozca la igualdad ante la ciudadanía" (MARSHALL, 2005, p. 19).

Es decir, la condición social no fue eliminada por el sistema social, la condición diferencial de clase es reemplaza por la condición uniforme de ciudadanía. La ciudadanía es una categoría clave como mediadora entre lo público y lo privado, le otorga sentido a la tarea y funciones del Estado, el ciudadano como individuo se re-

\footnotetext{
3 "Derechos civiles: compuesto por los derechos necesarios para la libertad individual de las personas. Libertad de palabra, pensamiento, derecho de poseer contrato y propiedad, derecho a la justicia, entre otros [...] Derechos políticos: derecho a participar en el ejercicio del poder político, derecho al sufragio universal [...]. Derechos sociales: variedad de derechos desde el bienestar económico y seguridad (distribución de la riqueza y la producción) hasta compartir plenamente la herencia social". (MARSHALL, 2005, p. 21, 22, 23).
}

EM PAUTA, Rio de Janeiro $-1^{\circ}$. Semestre de $2012-$ n. 29 , v. 10 , p. 101-118

Revista da Faculdade de Serviço Social da Universidade do Estado do Rio de Janeiro 


\section{heVibtg am pant?}

\} EMANCIPACIÓN POLÍTICA Y EMANCIPACIÓN HUMANA - CAPELO, M.; GIANNA, S. \}

conoce como sujeto político y se abstrae de sus verdaderas condiciones materiales de existencia a través de la interferencia mediación que el Estado imprime y ejerce en él.

\subsubsection{La emancipación social en Sousa Santos}

La segunda matriz teórica que influencia en el debate sobre la ciudadanía en el Trabajo Social es, como ya se adelantó, la obra de Sousa Santos. Para el autor, la sociedad contemporánea está atravesada por una "crisis y transición paradigmática y societal" que demanda una reinvención de la emancipación social.

Para el autor, "Cuando hablamos de reinventar la emancipación social, probablemente debamos usar la palabra en plural: emancipaciones sociales [...]". (SOUSA SANTOS, 2009, p. 20). La reinvención de la emancipación social tiene, para Sousa Santos (2006), dos necesidades, la primera, reconocer que la emancipación social es un concepto centralmente moderno, atravesado por una tensión propia de la modernidad entre la regulación y la emancipación y la segunda, que es ineludible seguir pensando la emancipación, sus posibilidades, pero no en términos propiamente modernos.

Estas consideraciones iniciales que realiza Santos sobre la emancipación social serían producto de la fase actual que atraviesa el capitalismo, su fase "desorganizada", donde no sólo se agotó el ideario de la modernidad, sino que también el mercado colonizó no sólo el espacio económico, sino también al Estado y a la comunidad entera (SOUSA SANTOS, 1998).

La colonización del mercado sobre las distintas esferas de la vida social, habría provocado la extinción de la "tensión creativa" desarrollada entre el capitalismo y la democracia. En la medida en que la democracia es absorbida por el mercado, esto provoca la restricción del poder de la democracia respecto a la redistribución social. Para Sousa Santos (2005) esto habría instaurado un "nuevo contrato social" en el "capitalismo desorganizado", que poco tiene que ver con la noción moderna de "contrato social". En palabras del propio autor,

Se trata, en primer lugar, de una contractualización liberal individualista, basada en la idea del contrato de derecho civil celebrado entre individuos y no en la idea de contrato social como agregación colectiva de intereses sociales divergentes. El Estado, a diferencia de lo que ocurre con el contrato social, tiene respecto a estos contratos de derecho civil una intervención mínima: asegurar su cumplimiento durante su vigencia sin poder alterar las condiciones o los términos de lo acordado. En segundo lugar la nueva contractualización no tiene, a diferencia del contrato social, estabilidad: puede ser denunciada en cualquier momento por cualquiera de las partes. Y no se trata de una opción de carácter radical sino más bien de una opción trivial. En tercer lugar, la contractualización liberal no reconoce el conflicto y la lucha como elementos estructurales del contrato (SOUSA SANTOS, 2005, p. 20-21). 


\section{ReVista em pgutti}

\} EMANCIPACIÓN POLÍTICA Y EMANCIPACIÓN HUMANA - CAPELO, M.; GIANNA, S. \}

Es decir, este "contrato individualista", que sería el imperante dentro de la fase actual del capitalismo, sería una degradación del contrato social regente de la sociedad. Si el contrato social siempre instauró límites entre la inclusión y la exclusión, entre quienes pertenecían al contrato y quienes no, el "contrato individualista" habría extremado los procesos de exclusión, restringido hasta el máximo la inclusión. Por lo tanto, la emancipación social sería el resultado de la creación de un nuevo contrato social, que sea inclusivo de aquellas exclusiones del contrato social moderno y que se base en

[...] una nueva teoría de la democracia que permita reconstruir el concepto de ciudadanía, una nueva teoría de subjetividad que permita reconstruir el concepto de sujeto y una nueva teoría de la emancipación que no sea más que el efecto teórico de las dos primeras teorías (SANTOS, 1998, p. 330).

Para reinventar la emancipación social es necesario recuperar y experimentar la "demo-diversidad", esto es, las diversas formas de participación democrática, al mismo tiempo que se instaura una nueva relación entre el Estado, el mercado y la comunidad. Dentro de las propuestas teóricas y políticas de Santos, se encuentra la "reinvención del Estado como novísimo movimiento social", considerando que este "nuevo Estado" deberá articularse con la comunidad, de forma tal de lograr algún tipo de control sobre el mercado. Es decir, se propone una emancipación social donde la comunidad sea el actor regente que junto al Estado regule el mercado.

Así, se vuelve central una forma de organización de la ciudadanía que no sea sólo vertical, entre el Estado y los ciudadanos sino también una relación horizontal entre los propios ciudadanos, donde la democracia no queda sólo reducida a una democracia representativa, sino que ésta se articula con modalidades de democracia participativa. Santos propone instaurar un nuevo constitucionalismo que represente estas transformaciones en la relación entre la comunidad y el Estado, de modo tal de superar el constitucionalismo moderno - basado en el acto libre de un pueblo que impone sus reglas a partir de un contrato social para vivir en paz dentro de un estado - y fundar un nuevo constitucionalismo, regido por la plurinacionalidad, la pluriculturalidad, la plurieticidad y la interculturalidad.

Este nuevo constitucionalismo, debe superar la universalidad de la ciudadanía y reconocer las diferencias y particularidades. En palabras de Santos:

Al consistir en derechos y deberes, la ciudadanía enriquece la subjetividad y le abre nuevo horizontes de autorrealización pero, por otro lado, lo hace por la vía de derechos y deberes generales y abstractos que reduce la individualidad a lo que hay de universal en ella, transforma los sujetos en unidades iguales e intercambiables en el interior de administraciones burocráticas públicas. La igualdad de la ciudadanía choca, así, con la diferencia de la subjetividad, tanto más cuanto que en el marco de la regulación liberal esa igualdad es pro- 


\section{heVibtg am pant?}

\} EMANCIPACIÓN POLÍTICA Y EMANCIPACIÓN HUMANA - CAPELO, M.; GIANNA, S. \}

fundamente selectiva y deja diferencias intactas, sobre todo las de la propiedad (1998, p. 292).

En síntesis, Sousa Santos propone una concepción de emancipación social que articule de un nuevo modo las relaciones entre el mercado, la comunidad y el Estado, de modo tal que sea posible una relación horizontal y vertical de estas instancias, donde una nueva concepción de ciudadanía, plural y diversa, con diversas instancias democráticas (representativas y participativas) logren controlar el mercado.

\subsubsection{Un análisis crítico desde la teoría de Marx}

El excurso precedente, intenta presentar de forma sintética algunos de los elementos y concepciones de Marshall y Sousa Santos en torno a la ciudadanía y la emancipación. A continuación, se pretende realizar algunos señalamientos críticos de las obras de estos autores, con el fin de mostrar los límites en dichas proposiciones teórico-políticas.

En primer lugar, debe destacarse la distinción existente entre Marshall y Sousa Santos en relación con Marx en lo referente al método de conocimiento. En ese sentido, la pregunta en torno a la función de la ciudadanía adopta posiciones distintas si se asume una perspectiva de totalidad o por el contrario, se parte de las ciencias sociales específicas. Así, el punto de partida de Marshall y Sousa Santos, desde una "sociología clásica o crítica/radical" según los casos, lleva a ambos autores a concebir la realidad como un conjunto de esferas autónomas, que entre sí tienen relaciones de exterioridad.

En el caso de Marshall, su posición parte de una relación de externalidad entre dos sistemas, la ciudadanía y las clases sociales, lo que lo lleva a una falsa contraposición entre el Estado político y sociedad civil (burguesa), cayendo en la ilusión de que el individuo se torna genérico mediante el estado, presentándose como natural la relación reificada entre el Estado político y la sociedad civil. Mientras que en Sousa Santos, la fragmentación de la realidad se presenta a partir de tres grandes esferas que la constituye: el Estado, el mercado y la comunidad. Estas esferas, también tendrían una relación de exterioridad entre sí, ya que el Estado apenas sería el espacio de las decisiones políticas, el mercado de las transacciones económicas y la comunidad el de las relaciones sociales. Si bien el autor no llega a plantear que estas esferas son plenamente autónomas, sí subyace esta división que no logra aprehender qué hay de político en las relaciones económicas, así como tampoco qué hay de económico y político en la comunidad y qué función cumplen cada una de estas esferas dentro de la sociedad capitalista.

A diferencia de estos autores, Marx puede aprehender de forma dinámica y contradictoria las mediaciones entre ciudadanía y clase social y entre mercado, comunidad y Estado, partiendo del hecho que estas esferas no son entes separados, sino que, por ejemplo, entre el mercado y la comunidad se generan relaciones privadas de producción, regidas por relaciones jurídicas y políticas, que son sostenidas y abaladas por el Estado. Como advierte Boron, hay una 


\section{ApVista all piuth}

\} EMANCIPACIÓN POLITICA Y EMANCIPACIÓN HUMANA - CAPELO, M.; GIANNA, S. \}

[...] imposibilidad de comprender la política al margen de una concepción totalizadora de la vida social, en donde se conjugaran y articularan economía, sociedad, cultural, ideología y política...la tensión entre la dinámica polarizante y excluyente de la sociedad civil, en realidad de la economía capitalista, y las pretensiones integradoras y universalistas del Estado burgués (2003, p. 304).

En segundo lugar, este elemento metodológico en común presente en las obras de Marshall y Sousa Santos les impide comprender la esencia de la relación entre ciudadanía y capitalismo: la propiedad privada de los medios de producción. Esta categoría, permite aprehender que la función de la emancipación política en el capitalismo es legitimar la apropiación privada del trabajo objetivado y del trabajo vivo.

En tercer lugar, esta relación de externalidad entre sistemas o esferas de la realidad lleva a que, por ejemplo, Sousa Santos señale que la problemática de la ciudadanía es su universalidad y por ende, su homogeneización que no permite reconocer las diferencias. Sin embargo, el problema central de la ciudadanía no es su universalidad, sino su alto grado de formalismo que impide la concreción real de la universalidad. Es decir, la democracia es un valor universal, no sólo por permitir una mediación entre el sujeto y el ser genérico, sino también porque ese universal, al superar las personificaciones formalizadoras de la ciudadanía, contienen en sí mismo lo singular, lo particular y diverso. Sólo mediante la superación de la democracia formal es posible una unidad de lo diverso entre lo singular y lo universal, entre el individuo y la comunidad de productores libres.

En cuarto lugar, estos autores, al no comprender el aspecto dialéctico entre la emancipación política y la emancipación humana consideran que la emancipación política plena puede sustentar por sí misma la "sociedad democrática", basada en una ciudadanía plena. Sin embargo, como advierte Borón (2006), se debe tener en cuenta que el término democrático, desde el surgimiento del modo de producción capitalista, ha quedado subordinado al capitalismo y que cuando se utiliza el concepto de democracia capitalista o burguesa, sería más correcto Ilamarla capitalismo democrático. En palabras del autor:

En la formulación capitalismo democrático queda claro que lo sustantivo es el capitalismo y que la democracia es una consideración adjetiva que no modifica sino superficialmente la estructura capitalista subyacente. En la formulación democracia capitalista, que no por casualidad es la que goza mayor predicamento en las ciencias sociales, el mensaje implícito es que lo sustantivo es la democracia siendo el capitalismo apenas una nota accidental que le otorga una tonalidad distintiva pero nada más. De ese modo se postula subliminalmente, que lo que cuenta es la sustancia democrática del orden social y no su fenomenología capitalista que, por eso mismo, no puede interferir 


\section{nevistg all paUt?}

\} EMANCIPACIÓN POLÍTICA Y EMANCIPACIÓN HUMANA - CAPELO, M.; GIANNA, S. \}

de ninguna manera con el funcionamiento de la estructura democrática de la sociedad. Así el capitalismo se mimetiza con la democracia. ¡Y quien podría estar en contra de esta! Se produce entonces una nada inocente inversión hegeliana, en donde el sujeto (el capitalismo) se convierte en predicado (la democracia) y ésta en sujeto. (Grifos do autor). (BORÓN, 2006, p. 17).

Lo que Marshall y Sousa Santos no problematizan, Marx lo hace, al abordar la contradicción irresoluble entre capitalismo y democracia. Para ello, se vuelve fundamental reconocer nuevamente que Marx conceptualiza a la realidad social desde una perspectiva de totalidad, la realidad es una totalidad concreta, dinámica y en movimiento. Desde ahí, capta las mediaciones existentes entre el modo de producción capitalista y la necesidad de la emancipación política, concluyendo que toda relación económica (contenido), que se realiza de modo indirecto mediante el cambio de las mercancías, tiene por forma una relación jurídica privada (contrato de compra-venta) y una relación jurídica pública (relaciones de ciudadanía), que en la apariencia se presenta como relaciones directas entre poseedores de mercancías.

En síntesis, la perspectiva de totalidad le permite a Marx captar no sólo las mediaciones, sino también las formas en que se desarrolla su contenido. Es decir, si Marx se hubiera quedado en el simple plano de la circulación, habría llegado a la conclusión que el intercambio es posible por el derecho, mientras que asumiendo una mirada de totalidad, reconoce que el contenido (las relaciones sociales y económicas de producción) se realiza en formas (relaciones jurídicas) que muchas veces son "contrarias" y "opuestas" al contenido.

\section{Trabajo Social y ciudadanía: una primera aproximación}

\section{a sus implicancias y características en el Trabajo Social argentino}

A partir del desarrollo expuesto anteriormente, es posible afirmar que tanto la emancipación política como la ciudadanía son dos categorías centrales para pensar y reflexionar en torno al Trabajo Social, así como en los límites, posibilidades y horizontes presentes en la intervención profesional.

Conlleva este análisis, la incorporación de por lo menos tres momentos históricos relevantes que atraviesan la profesión, ligados al movimiento de luchas de clases que se fue presentando en el país, y que incidieron directamente en retrocesos y avances del colectivo profesional. Estos tres momentos (sucintamente) se pueden enumerar de la siguiente forma: la dictadura militar (1976-1983), la vuelta de la democracia con su implementación del modelo neoliberal (1983-2001) y la década que continúa a la crisis del 2001-2002, en la cual a partir de un auge en la insurrección popular los gobiernos venideros se ven obligados a cuestionar las políticas neoliberales e implementar políticas de corte progresista.

Esta relación entre la emancipación política, la ciudadanía y el Trabajo Social argentino se expresa en diversas esferas de la profesión. En primer lugar, en su re- 


\section{nevistg am pguth}

\} EMANCIPACIÓN POLITICA Y EMANCIPACIÓN HUMANA - CAPELO, M.; GIANNA, S. \}

glamentación jurídica, objetivada en una Ley Profesional ${ }^{4}$, un Código de Ética ${ }^{5}$ y sus Incumbencias Profesionales ${ }^{6}$ (específicamente del Colegio Provincial de la Provincia de Buenos Aires).

En el plano normativo jurídico no aparece el concepto o término de ciudadano o ciudadanía, pero si una concepción o enunciado de un sujeto sobre el que se debe trabajar su conducta para ser integrado, esto es, una concepción de individuo abstracto, de cariz positivista, donde la sociedad es una simple sumatoria de los miembros que la componen, los derechos enunciados bajo el derecho, que si bien necesariamente es planteado en un término formal, al colocarlo de manera genérica se lo condensa de toda la totalidad que pudiera abarcar el ser social. Se analiza a los individuos en tanto inmersos en un sistema de "relaciones de igualdad", en donde a partir del intercambio son libres y formalmente iguales, y ante cualquier situación que conlleve a la alteración de esa armonía, se debe apelar a la autoridad de la sociedad, abordando los problemas - en termino de disfuncionalidades - a partir de una normatividad social basada en la solidaridad y la integración.

En segundo lugar, en los ámbitos de formación profesional, los contenidos curriculares de las diversas carreras incorporan como contenido la cuestión de la ciudadanía, pero no como parte de las materias pertenecientes al área o al trayecto de Trabajo Social. Mediante el relevamiento en torno a las temáticas de los Congresos Nacionales organizados por la Federación Argentina de Asociaciones Profesionales de Servicio Social (FAAPSS) y las producciones escritas en forma de artículos de revistas y libros, la temática de la ciudadanía fue incorporada aproximadamente hace veinte años ${ }^{7}$.

Con respecto a los debates rastreados en congresos y encuentros si bien hay un cuestionamiento al concepto neoliberal que aborda la ciudadanía en términos de sujeto de consumidor, se interpela al mismo principalmente teniendo como referencia a la obra de Marshall y ya en los últimos años (post 2001) en dicha interlocución analítica-teórica se incorporan las producciones contemporáneas de Sousa Santos. Cabe aclarar, y esto es muy significativo, que en ninguna de las producciones recuperadas o analizadas se explicita o emerge la matriz marxiana como referencia teórica. Con lo cual, si bien distintos sectores del colectivo profesional asumen como horizonte para el Trabajo Social argentino la cuestión de la ciudadanía, en términos de concebir al usuario sólo como sujeto de derecho, esta posición unilateral evidencia la ausencia de una matriz critico-dialéctica que permita captar el significado contradictoria de la misma en el marco de la sociabilidad capitalista.

\footnotetext{
${ }^{4}$ Ley 10751 El ejercicio de la profesión de Asistente Social en al jurisdicción de la Provincia de Buenos Aires queda sometido a las disposiciones de la presente Ley. Promulgada en diciembre de 1988.

${ }^{5}$ Código de ética regido y creado a partir de la ley 10751. Art. 51: Las normas de este código rigen la actividad de los profesionales en Servicio Social, matriculados en la Provincia de Buenos Aires, en toda su extensión y ninguna circunstancia eximirá de aplicarlas salvo causa justa a criterio del Tribunal de Disciplina.

6 Incumbencias profesionales reglamentadas por el ministerio de Educación y Justicia de la Nación. Resolución ${ }^{6}$ Incumbenci $579 / 86$.

n. 579/86.
7 Del rastreo bibliográfico realizado hasta el momento, recién en 1978 se encontró el primer trabajo que alude explícitamente a la ciudadanía y ya a partir de la década del ochenta y noventa los debates en torno a la ciudadanía aunque exiguos, comenzaron a ser recurrentes dentro de la profesión.
}

EM PAUTA, Rio de Janeiro - $1^{\circ}$. Semestre de $2012-$ n. 29 , v. 10, p. 101-118

Revista da Faculdade de Serviço Social da Universidade do Estado do Rio de Janeiro 


\section{heVibtg am pant?}

\} EMANCIPACIÓN POLÍTICA Y EMANCIPACIÓN HUMANA - CAPELO, M.; GIANNA, S. \}

En tercer lugar, diversos componentes de la ciudadanía y la emancipación política se expresan en el cotidiano de la intervención profesional, como por ejemplo en la participación de los sujetos en tanto ciudadanos en la esfera pública y estatal, las demandas de la población usuaria en la institución que exigen la ampliación de las prestaciones y recursos, las modalidades de atención institucionalizadas por las organizaciones empleadoras del trabajador social, son apenas algunas de las expresiones cotidianas que ligan al Trabajo Social con la ciudadanía. También, esto se enuncia cuando el Trabajo Social aborda diversas necesidades y problemas sociales expresados por sujetos colectivos y la intervención liga las mismas con un derecho.

Se podría plantear que por la naturaleza de la intervención del trabajador social se trabaja con la problemática de la emancipación política y la ciudadanía, que el profesional, a partir de múltiples mediaciones, se enfrenta - encuentra desde un carácter puntual y directo con el individuo que demanda, aunque determinado por el Estado y mediado tanto a través de un contrato de trabajo, en su carácter de asalariado y por las políticas sociales, que ofician de instrumentos imprescindibles para responder o abordar los problemas en cuestión.

Estas particularidades, le confiere al trabajador social una posición contradictoria: los problemas son de orden estructural-social aunque se manifiestan de manera individual. De este modo, la intervención estará velada, mostrándose de un modo mistificado, entendiendo que tal situación trasciende a la profesión debido a que la realidad, en tanto proceso histórico de determinadas relaciones sociales, en este caso las relaciones sociales que tienen un carácter burgués, se presentan mistificadas, dando cuenta de procesos de reificación, presentándose el capital como cosa y no como una relación social de explotación y antagonismo. Dicha reificación de la realidad tendrá un carácter totalizador y universalizante.

Así, el trabajador social que pueda críticamente analizar y develar el carácter contradictorio del ciudadano y el hombre, podrá también develar su propia práctica. Dicho de otra manera, en la propia práctica social se observan expresiones de esta tensión interior y el movimiento que la contiene la encubre al mismo tiempo. Esto se vislumbra, por ejemplo, en cómo es presentada la demanda por el sujeto de la acción profesional, una demanda de carácter social producto de un proceso de lucha y conflicto se presenta como algo puntual, práctico-concreto (de carácter útil), individual y a su vez la misma - la intervención - sólo se realiza o materializa como tal, si se afronta en forma directa el problema puntual que le es manifestado.

Con lo cual, el Trabajo Social para desplegar su práctica, en tanto ejercicio profesional, reproduce necesariamente un movimiento contradictorio (propio de la practica social), debiendo negar el fenómeno para afirmarlo, en otras palabras, solo puede asumir el problema individual negándolo en su singularidad para afirmarlo en lo social. Esta práctica, como ya fue planteado, no es aislada ni se produce por fuera del propio movimiento y dinámica de la propia realidad, reproduce una práctica social pero expuesta y expresada en su propia particularidad.

La tensión permanente con la que se encuentra el Trabajo Social, lo individual y lo social, constituyen una unidad y a su vez una contraposición, enfrentando el problema social en lo individual, que en su devenir se manifiesta, denuncia y niega 


\section{nevistg am pguth}

\} EMANCIPACIÓN POLITICA Y EMANCIPACIÓN HUMANA - CAPELO, M.; GIANNA, S. \}

a sí mismo, y es así, como en el síntoma se denuncia lo social que porta lo individual. Es pertinente aquí colocar el concepto de síntoma social ${ }^{8}$, que remite directamente al conflicto irresoluble y estructural del modo de producción capitalista, irresoluble dentro de las posibilidades del propio sistema, estructural en cuanto a su carácter totalizador y universalizante, un síntoma emergente, que no se muestra como tal, que se encarna de una manera mistificada y fenoménica en el individuo, responsabilizándolo a sí mismo, de las diferentes situaciones sociales por las cuales es atravesado.

Analizar críticamente la realidad de los sujetos con los cuales se trabaja, en tanto sujetos de derechos, pero situados en su propia condición de clase, dará luz a estos procesos necesarios de desmitificar la intervención. Son momentos dialécticos del propio proceso de la práctica social-profesional, desmitificar la intervención es desmitificar el carácter absoluto y fragmentario de ser ciudadano, es reconocer las propias contradicciones, realizando lecturas y abordajes posibles dentro de la realidad que acompañen procesos emancipatorios no sólo en su dimensión política sino también humana.

\section{Consideraciones finales}

Finalmente, ciertas posiciones teóricas incorporan la emancipación política planteada por Marx pero no incorporan en su análisis - si bien puede estar enunciada - la cuestión de la emancipación humana, quizás una de las categorías centrales del texto de Marx, que propone que la lucha del hombre por apropiarse de su ser genérico, no es más que la superación de las personificaciones (los derechos), y así re-apropiarse del trabajo y de los productos del mismo.

En otras palabras, Marx - al plantear la noción de emancipación humana reconoce que es mediante la apropiación de su ser genérico y de la naturaleza donde realmente puede transformar su realidad. Por su parte, una mirada reformista propone que las modificaciones en la sociedad democrática se producirían en la esfera de los derechos - como derechos del hombre - a partir de su emancipación política y que esta alcanzaría para modificar la realidad, negando así la lucha de clases.

Desde aquí, es imprescindible para el Trabajo Social poder asumir y apropiarse de estos debates, en términos no solo políticos sino también teórico-metodológicos, dando cuenta de las mediaciones necesarias para poder intervenir de manera crítica en la realidad en la cual se actúa. Al mismo tiempo, es Ilamativa la ausencia de la perspectiva marxista en este debate y es por ello, que este artículo intenta presentar los primeros avances en torno a esta temática, la dialéctica de la emancipación política y la emancipación humana y su mediación con el Trabajo Social.

\footnotetext{
8 "El antagonismo puesto en evidencia al analizar el Modo de Producción Capitalista que acontece con la instauración de una estructura universal que todo torna intercambiable y donde todo tiene destino de mercado, caracterizará el escenario donde nosotros situaremos nuestra definición de síntoma social [...] La producción universal genera en su producto (la mercancía) un tipo particular que niega su propia condición; la equivalencia universal no es equivalencia universal. Allí, en ese preciso lugar, se genera un síntoma; un producto particular, inherente a la estructura, niega su atributo universal (Zizek); aquello que instaura el sistema, al mismo tiempo produce aquello que lo invalida [...]." (LÓPEZ, 2010, p. 13).
} 


\section{nevista am pantg}

\} EMANCIPACIÓN POLÍTICA Y EMANCIPACIÓN HUMANA - CAPELO, M.; GIANNA, S. \}

\section{Referências}

BORÓN, A. Estado, capitalismo y democracia en América Latina. Buenos Aires: CLACSO, 2003.

Crisis de las democracias y movimientos sociales en América Latina: notas para la discusión Revista OSAL. Buenos Aires: CLACSO, n. 20, p. 12-20, may-ago 2006.

COUTINHO, C. N. Contra a corrente: ensaios sobre democracia e socialismo. Sao Pablo: Cortez Editora, 2000.

IASI, M. Ensaios sobre conciência e emancipação. Sao Paulo: Expressão Popular, 2007.

LÓPEZ, A. Ética, política y síntoma en Salud Mental. Hegel, Marx, Lacan. Dossier científico "Lo singular, lo colectivo". La Plata: Colegio de Psicólogos de la provincia de Buenos Aires, año III, n. 3, p.11-15, 2010.

LUKÁCS, G. Lenin-Marx. Buenos Aires: Editorial Gorla, 2005.

MARSHALL, T. H. Ciudadanía y clase social. Buenos Aires: Editorial Losada, 2005.

MARX, K. Elementos fundamentales para la crítica de la economía política (Borrador 1857-1858). (Grundrisse). Buenos Aires: Siglo Veintiuno Editores, 1971.

Escritos de juventud. Buenos Aires: Editorial Antídoto, 2006.

Escritos de juventud sobre el derecho. Textos 1837-1847. Madrid: Anthropos Editorial, 2008.

SOUSA SANTOS, B. De la mano de Alicia: lo social y lo político en la postmodernidad. Cali: Ediciones Uniandres, 1998. 2005.

Reinventar la democracia, reinventar el Estado. Buenos Aires: CLACSO,

Renovar la teoría crítica y reinventar la emancipación social. Buenos Aires: CLACSO, 2006.

Pensar el Estado y la sociedad: desafíos actuales. Buenos Aires: CLACSOWaldhuter editores, 2009.

Recebido em 07 de julho de 2012.

Aceito para publicação em 21 de agosto de 2012. 